\title{
Infant Spontaneous Motor Tempo
}

\section{Sinead Rocha ${ }^{1,2} \odot$ | Victoria Southgate ${ }^{3}$ | Denis Mareschal ${ }^{1}$}

\author{
${ }^{1}$ Centre for Brain and Cognitive \\ Development, Birkbeck University of \\ London, London, UK \\ ${ }^{2}$ Centre for Neuroscience in Education, \\ University of Cambridge, Cambridge, UK \\ ${ }^{3}$ Department of Psychology, University of \\ Copenhagen, Copenhagen, Denmark \\ Correspondence \\ Sinead Rocha, Centre for Brain and \\ Cognitive Development, Birkbeck \\ University of London, London, UK. \\ Email: sineadrocha@gmail.com \\ Funding information \\ Economic and Social Research Council, \\ Grant/Award Number: Studentship to SR
}

\begin{abstract}
Spontaneous Motor Tempo (SMT) is influenced by individual differences in age and body size. We present the first data documenting the SMT of infants from 5 to 37 months of age using a simple drumming task. As in late childhood and adulthood, we predicted that infant SMT would slow across the first years of life. However, we find that older infants drum more quickly than younger infants. Furthermore, studies of adults suggest larger bodies prefer slower rhythms. This relationship may be the product of biomechanical resonance, or effects may be driven by rhythmic experience, such as of locomotion. We used infants, whose body size is dissociated from their predominant experience of locomotion as their parent often carries them, to test this argument. We reveal that infant SMT is predicted by parent, but not own, body size, supporting a passive experience-based argument, and propose that early rhythm may be set by repetitive vestibular stimulation when carried by the caregiver.

KEYWORDS

body size, infant development, locomotion, rhythm, Spontaneous Motor Tempo, vestibular
\end{abstract}

\section{1 | INTRODUCTION}

Humans are highly rhythmic animals, with rich beat perception and synchronization capabilities that set us apart from other species (Iversen, 2016). There are remarkable universals in the music produced across disparate cultures, including a propensity for a regular, isochronous, beat (Savage, Brown, Sakai, \& Currie, 2015). The ontogeny of our rhythmic behaviors has been the focus of much recent research, and can provide vital clues to the evolution of musical skill (see Ravignani, Honing, \& Kotz, 2017). One of the simplest rhythmic capacities is the ability to produce a regular beat. Understanding the development of Spontaneous Motor Tempo (SMT), or our natural rate of rhythmic movement (Fraisse, 1982), provides the opportunity to better understand the roots of our rhythmic predispositions.

Spontaneous Motor Tempo is often measured as the inter-onset interval (IOI) between a person's self-paced finger taps (Fraisse,
1982). Adult SMT is highly stable, showing little intra-individual variability across testing sessions spanning several days (Vanneste, Pouthas, \& Wearden, 2001). However, across the lifespan, SMT is known to change. Children's SMT is faster than adults, who are in turn faster than older people, with a cubic relationship suggesting that SMT slows with age during childhood and late adulthood, but remains consistent through mid-adulthood (aged 18-38 years, $M=630$ ms IOI; McAuley, Jones, Holub, Johnston, \& Miller, 2006). The earliest measures of SMT come from Provasi and Bobin-Bègue (2003), who demonstrate that reliable spontaneous tapping data can be obtained from 2.5-year-olds, with toddlers tapping significantly faster than adult controls, at around $400 \mathrm{~ms} \mathrm{IOI}$, but with no difference in SMT between 30 and 54 months of age.

It has been widely reported that both adult SMT and adult walking cadence lie in the range of $600 \mathrm{~ms} \mathrm{IOI}$, or around 120 beats per minute (Fraisse, 1982). One possibility is that this relationship is causal, with walking cadence being the origin of our

This is an open access article under the terms of the Creative Commons Attribution License, which permits use, distribution and reproduction in any medium, provided the original work is properly cited. 
preferred tempo at which to move, and perhaps even to listen. Studies revealing correlations between anthropometrics (measures of body size) and SMT have been used to argue for this possibility, following the logic that body size may be used as a proxy for walking cadence, as rate of locomotion should be set by the mechanics of the human body (see Repp, 2007; Todd \& Lee, 2007, for debate on this subject). Anthropometrics correlate with SMT (Mishima, 1965), naturalistic full body dancing (Dahl, Huron, Brod, \& Altenmüller, 2014), and preferred tempo in a perceptual task (Todd, Cousins, \& Lee, 2007). A critical issue with this argument is that within-subject correlational studies cannot show a causal impact of walking cadence on SMT: results correlating body size with the tempo of motor tasks (Dahl et al., 2014; Mishima, 1965) may rather support a broader concept of biomechanical resonance, or a natural frequency of movement across the body. Crucially, however, the correlation with preferred auditory tempo (Todd et al., 2007) suggests that some experience must be necessary for rhythmic bias, as there is unlikely to be a genetic mechanism that directly matches body size to auditory preferences (Trainor, 2007). Locomotion is a sensible, pervasive, candidate experience for such a transfer (Todd et al., 2007; Trainor, 2007).

Infants present a unique opportunity for understanding the impact of walking cadence, removed from the context of one's own body size. There is strong evidence that vestibular movement to an ambiguous beat biases the perception of a rhythm in infancy (Phillips-Silver \& Trainor, 2005). Before an infant is able to efficiently locomote herself, she experiences months of vestibular stimulation while being carried by her caregiver. There is, therefore, a clear dissociation between infant body size and the rate of predominant (vestibular) locomotive experience, which may be reflected in the parents' body size. The current study aims to exploit this dissociation to test the hypothesis that locomotive experience drives our basic rhythmic preferences.

Although infant SMT has not previously been measured, infants are known to spend up to $40 \%$ of their time performing repetitive movements (Thelen, 1979, 1981). Infants from as young as 4 months of age have been shown to spontaneously move rhythmically in the context of music (Fujii et al., 2014; Ilari, 2015; Zentner \& Eerola, 2010). Ten- and 18-month-olds engage in rhythmic movement with small instruments (Rocha \& Mareschal, 2017). Thus, while traditional tapping tasks may lie outside the range of infant motor control, it seems plausible to measure infant SMT using a more gross motor rhythmic movement. The current study does so using a drumming paradigm, which allows for (a) a large surface area for infants to strike; (b) a unimanual or bimanual whole hand action; and (c) rewarding auditory feedback from hits, motivating infants to make repeated movements.

The current study, thus aims to uncover the rate of spontaneous rhythmic movements in infants, who have little to no experience of locomoting themselves, but rich experience of being carried by a locomoting adult. In line with findings from across the lifespan, we predict that infant SMT will slow with age. There are two hypotheses for the relationship between infant SMT and

\section{Research Highlights}

- We provide a novel behavioral measure of infants' natural rate of rhythmic movement, termed Spontaneous Motor Tempo (SMT)

- Infant SMT can be measured from 5 months of age using a simple drumming task

- Infant drumming becomes faster and more regular with age. Tempo and regularity also correlate independently of age.

- Infant SMT is related to parent body size, but not own body size.

anthropometric measures. If infant SMT is related to own body size, this would support a biomechanical resonance explanation, as infants' own walking experience is unlikely to be regular, or pervasive, enough to provide a tempo for other actions. However, if infant SMT is related to parental body size, this would instead suggest that information gained from the passive experience of locomotion drives SMT.

\section{2 | METHOD}

\section{1 | Participants}

An opportunity sample of 115 infants (48 females, mean age $=11.7$ months, $S D=6.87$ months, range $=4$ to 37 months) took part in this study. An additional 66 infants were recruited but excluded for not providing sufficient drumming data, see criteria below. Infants were recruited to take part in the study at a public science event; the attrition rate partly reflects that infants were able to leave the testing area for other activities if they so desired. The Departmental ethics committee granted ethical approval. All caregivers gave written, informed consent concerning the experimental procedure. Infants received a certificate as a thank you for participation.

\section{2 | Procedure}

Testing took place inside a blackout tent located in the foyer of a children's theatre. Infants were sat on their caregiver's lap or on the floor in front of their caregiver, within easy reach of the drum table. The experimenter sat adjacent to the drum table and opposite the infant. The experimenter demonstrated that the drum made a sound by hitting the drum with the flat of her hand once, and prompting the infant to drum using verbal cues such as 'Shall we play with the drum?', 'Can you hit the drum?', or 'What noise does the drum make?'. This prompt was repeated as necessary throughout the session, but with a minimum interval of $2 \mathrm{~s}$ 
between prompts to ensure the experimenter did not provide a rhythmic example. Infants were allowed to move freely and engage with the drum as they wished, but were kept engaged by the experimenter through eye contact and smiles, and were verbally encouraged to hit the drum if they were not doing so spontaneously. Between bouts of drumming, the experimenter interacted with the child by saying 'Well done, you're doing a great job!', and encouraged them to continue ('Shall we play some more with the drum? What does it do?'), regardless of their behavior during the trial. Infants interacted with the drum for an average of $2 \mathrm{~min} 20 \mathrm{~s}$ $(S D=1 \mathrm{~min}, 1 \mathrm{~s})$, with the trial terminated if the infant became bored or fussy, or the infant had drummed for more than $2 \mathrm{~min}$ continuously.

Either before or after infant participation, caregivers completed a short questionnaire about their infant's gross motor skills and milestones and experience of being carried. To ascertain infant and parent anthropometrics, a second experimenter administered the questionnaire and took measurements of infant and caregiver limb length (arm length, leg length), and asked the caregiver to self-report their height. Arm length was calculated by adding measurements from the spine to the shoulder to measurements from the shoulder to the wrist. Leg length was measured from the hipbone protrusion to the ankle. All adults were measured in a standing position. Infants were measured when standing (if able to hold themselves in a standing position), lying supine on the floor, or while being held by the caregiver.

\section{3 | Apparatus}

The drum was a 12-inch tuneable wood shell and natural skin head drum, secured to a height and angle adjustable mini-table. Sound was recorded from the drum via a Piezo contact microphone pickup attached with adhesive tape to the underside of the drum shell, and connected to a Focusrite Scarlett 2i2 (American Music and Sound), a hardware interface connecting the microphone audio signal to the computer (MacBook Pro; Retina, 15 -inch, Mid 2014). The Scarlett 2i2 was selected as the audio input and the sound recording was taken using Audacity ${ }^{\circledR}$, version 2.1.2 (2015). ScreenFlow (Telestream, Inc.) was used to create a simultaneous screen capture of the Audacity recording and video footage of the infant using the forward facing built-in webcam.

\section{4 | Data processing}

The Screenflow captures of the testing sessions were used to code all incidences where infants hit the drum with one or both of their hands. The corresponding time point of the audio signal was then identified and the experimenter hand marked the onset of each hit (as defined by the first peak in the sound stream, see Figure 1 for example). For each 'bout' of drumming (i.e., series of hits), the time stamp of each hit onset was recorded, along with how many hits

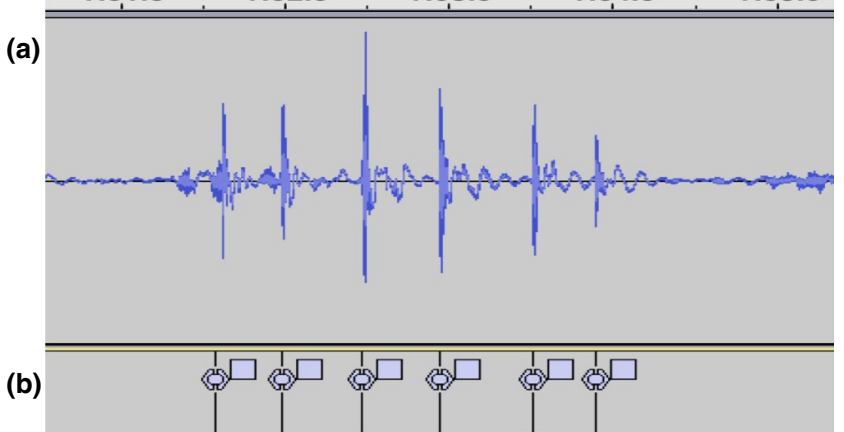

FIGURE 1 (a) Example of the audio signal produced by a bout of infant drumming. (b) The corresponding hand-coded event markers for the onset of each drum hit

were in the bout, and whether the bout was produced by one hand drumming, both hands drumming simultaneously, or both hands in an alternating sequence. An independent researcher blind to the aims of the study double coded the video data for 30 infants. The single-measure ICC for the IOI was .924 , with a $95 \%$ confidence interval from .790 to $.968(F(29,29)=33.353, p<.001)$.

To best match the adult literature on unimanual tapping, the following analyses were performed on the IOI of unimanual hits, or on the IOI of the first hand to strike during bimanual hits, with alternating sequences excluded. Data where the onset of the hit was ambiguous (i.e., because of wire noise, very low-amplitude hitting, etc.) were also discarded. Matlab (MATLAB R2015b; The MathWorks Inc.) was used to calculate the IOI. The mean IOI was calculated for each participant and taken as a measure of SMT. The relative standard deviation (RSD - also known as the coefficient of variation; the ratio of the standard deviation to the mean, expressed as a percentage) of the IOI was also calculated for each participant and taken as a measure of regularity, that is, a low relative standard deviation indicated more consistent drumming. To be included as a 'bout', infants had to perform four sequential hits with no more than a 2 s IOI between hits (in line with Fraisse, 1982). Infants who did not have at least one such 'bout' were excluded from further analyses, reducing the final sample to 115 infants. Eleven infants did not make any hits, and a further 55 made singular hits but not 'bouts'. The median number of hits produced by the final 115 infants was 19, ranging from 4 to 139 . The number of valid hits produced by the infant does not correlate with the core measures of tempo and regularity of drumming (all $p>.05$ ). In order to test whether the amount of valid data contributes to our core measures two linear regressions were run with mean IOI and RSD as independent variables, and age in days and number of drum hits as dependent variables. There was no contribution of number of hits to drumming tempo (overall model for mean IOI; $\left[F(2,99)=8.646, p=.0003, R^{2}=.1487\right]$; infant age $[\beta=-2.835 \mathrm{e}-04, t(99)=-3.819, p=.0002]$; number of hits $[\beta=-4.325 \mathrm{e}-04, t(99)=-.759, p=.450])$, or drumming regularity (overall model for $\operatorname{RSD}\left[F(2,99)=3.880, p=.024, R^{2}=.073\right]$; infant age $[\beta=-0.016283, t(99)=-2.72, p=.008]$; number of hits $[\beta=-0.054, t(99)=1.181, p=.241])$. Data S1 shows the distribution 
of the amount of drumming performed by each infant and the (lack of) relationship between number of drum hits and infant SMT.

\subsection{Statistical analyses}

Data were processed as described above and descriptive statistics on our measures of tempo and regularity were taken, and correlated with age, in order to describe the full sample. Linear regressions on the questionnaire data were performed. When looking at the descriptive statistics of infant regularity (RSD), it became very clear that some infants were drumming very erratically. Adult participants can be asked to tap at a regular rate, but we could not give the participants instruction to drum regularly. Consequently, in order to define infant drumming as reflective of an underlying SMT, we had to apply a criterion of regularity. While we, therefore, first describe the full response of valid infant drumming $(n=115)$, we made a post hoc decision to remove drummers who had an RSD 1 SD above the group mean, the equivalent to an RSD of over 35 , before characterizing infant SMT. Linear regression analyses test the contribution of infant and parent factors to infant SMT. Frequentist statistics are supplemented by Bayes Factors, such that both accepting and rejecting the null hypotheses are possible (see, e.g., Dienes, 2014, advocating for this approach). Plots were created in R 4.0.0 (2020) using ggplot2 (Wickham, 2016).

\section{$3 \mid$ RESULTS}

We hypothesized that infant drumming would slow with age. Across all participants, the mean tempo was 542 ms, with a standard deviation of $160 \mathrm{~ms}$. Contrary to our hypothesis, a two-tailed Pearson correlation shows that tempo is negatively correlated with age, such that older infants are faster $(r(114)=-.279, p=.003$; see Figure $2 \mathrm{~A})$. Older infants' drumming is also characterized by a lower relative standard deviation $(r(114)=-.217, p=.021$; see Figure $2 \mathrm{~B})$, and regularity and tempo are correlated independently of age $(r(114)=.509$, $p<.001$ ). We do not find differences in tempo (Data S1) or regularity (Data S1) due to time spent in different motoric activities.

Due to the large variability in infant drumming, and considering that the criterion of the regularity of the action is essential to define SMT, infants who drummed sporadically were excluded from further analyses. Within this subsample of 93 remaining participants (37 females, mean age $=12.3$ months, $S D=7.13$ months, range $=5-37$ months), the mean tempo is $504 \mathrm{~ms}$ with a standard deviation of $112 \mathrm{~ms}$. We no longer see a relationship between infant age and tempo $(r(92)=-.165, p=.113)$ or regularity $(r(92)=$ $-.115, p=.272$ ), although the correlation between age and regularity is maintained, such that faster SMT is related to less variability $(r(92)$ $=.266, p=.009$ ). As before, we do not find differences in tempo or regularity due to time spent in different motoric activities (all $p>$.05)

Of primary interest was whether infant SMT might correlate with own body size, suggesting biomechanical resonance; or whether infant SMT would correlate with parental body size, suggesting a role for passive experience. A linear regression with SMT as the dependent variable was performed on the remaining infants, with Infant Age, Arm Length, and Leg Length and Parent Height, Arm Length, and Leg Length entered as predictors. Degrees of freedom in the following analyses reflect the number of infants for whom all infant and parent measures were taken. The resulting model was significant, $\left(F(6,55)=3.321, p=.007, R^{2}=.266\right)$, explaining $26.6 \%$ of variance of the dependent variable. Infant Age $(\beta=-.459, t(55)=-2.596$, $p=.012)$ and Parent Height $(\beta=.413, t(55)=2.577, p=.013)$ were significant predictors of SMT. In order to further ascertain whether the predictors provided evidence for either the alternative hypothesis, a contribution of anthropometrics to infant SMT, or for the null hypothesis, no contribution of the measurements to SMT, we used JASP (JASP Team 2017; Version 0.8.1.2) to calculate the Bayes Factors for each predictor, using the default priors. Table 1 contains (a)

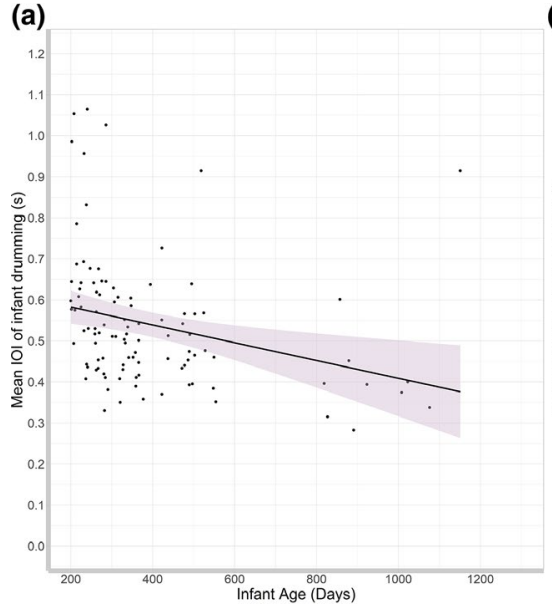

(b)

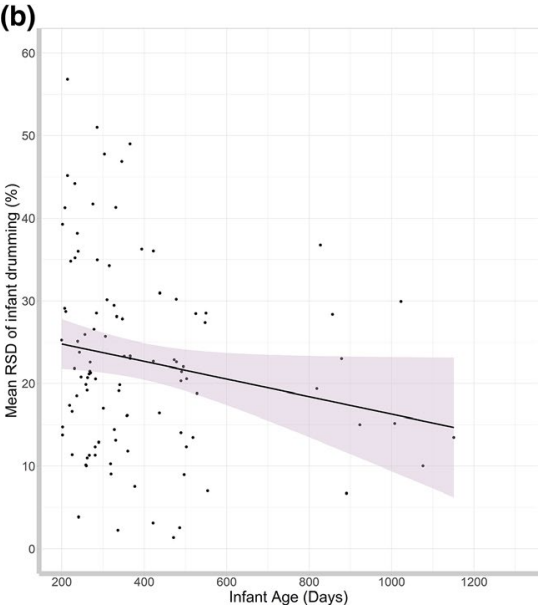

FIGURE 2 (a) Scatterplot of the relationship between infant age and rate of infant drumming (mean inter-onset interval [IOI] of drum hits). (b) Scatterplot of the relationship between infant age and regularity of infant drumming (relative standard deviation of the IOI of drum hits) 
TAB LE 1 Linear Regression Coefficients and Bayes Factors for effects of infant age and infant and parent anthropometrics on infant SMT of the final sample.

\begin{tabular}{|llrlr|} 
& $\begin{array}{l}\text { Standardized } \\
\text { beta }\end{array}$ & \multicolumn{1}{l}{$\boldsymbol{t}$} & \multicolumn{1}{l}{$\boldsymbol{l}$} & \multicolumn{1}{c|}{$\mathrm{BF}_{10}$} \\
\hline Infant Age & 0.580 & 0.580 & .012 & 10.061 \\
\hline Infant Leg Length & .003 & .016 & .988 & 0.839 \\
\hline Infant Arm Length & .238 & 1.303 & .198 & 0.474 \\
\hline Parent Height & .413 & 2.577 & .013 & 6.179 \\
\hline Parent Leg Length & -.081 & -.519 & .606 & 0.580 \\
\hline Parent Arm Length & -.168 & -1.305 & .197 & 0.290 \\
\hline
\end{tabular}

the standardized coefficients and Bayes Factors for all predictors. Bayes Factors over 3 or under 1/3 represent substantial evidence for the alternative/null, respectively, with values close to 1 representing weak or anecdotal evidence (Dienes, 2014). We find good evidence for the contribution of Infant Age $\left(\mathrm{BF}_{10}=10.061\right)$, suggesting that these data are more than 10 times more likely to be observed under the hypothesis than the null; and Parent Height $\left(\mathrm{BF}_{10}=6.179\right)$, showing over six times the evidence for an effect of parental height on infant SMT. For all other measures, the Bayes Factors suggest our data are more likely to be observed under the null (no contribution to infant SMT), although this evidence is weak for all measures except Parent Arm Length, where we see more than three times the evidence for the null hypothesis (Parent Arm Length $\mathrm{BF}_{10}=0.290$ ). Parent height was not correlated with infant arm length (two-tailed Pearson correlation, $[r(65)=-.068, p=.593]$ ) or infant leg length $(r(66)=.031, p=.804)$.

Finally, in order to provide an exploratory estimate of the within-subject stability of infant SMT, the IOI of two independent bouts of drumming per infant were correlated (data taken from 38 infants who produced two bouts, bout length ranged from 4 to 59 hits), and reveal a highly significant correlation $(r(38)=.601, p<.0001)$. A paired samples $t$ test confirms there is no statistical difference between the mean IOI of bout 1 and bout 2 within this subsample $(t(38)=-1.339, p=.189, \mathrm{BF}=.394)$, although Bayes Factors show only anecdotal to moderate evidence for the null hypothesis of no difference in SMT between trials.

\section{DISCUSSION}

Infant drumming becomes faster and more regular with age, and we provide evidence that infant SMT is predicted by infant age and parental height. We contend that infant SMT is related to both the infants' own ability to make repeated, targeted, movements, and potentially, the cadence of the parent's walking, which they experience passively while being carried.

We were able to successfully collect data using a simple task, effectively engaging infants as young as 5 months old through to walking, talking pre-schoolers, and providing a seemingly stable measure of SMT. That the rate of infant drumming in our full sample negatively correlates with age is at first glance at odds with the existing developmental literature. From childhood to late adulthood, SMT is known to slow with age (McAuley et al., 2006), although it is not shown to change between 18 and 54 months (Bobin-Bègue \& Provasi, 2008; Provasi \& Bobin-Bègue, 2003). While studies have not previously investigated the timing of repetitive movements in the first year of life, our results are consistent with the fact that infants become better at controlling their movements (Goldfield, 1995), and it is likely that infant performance on our task at least in part reflects the infant's level of motor coordination, with movements becoming faster as motor control increases. Within the subsample of infants who drummed rhythmically enough to indicate their SMT, our results align with findings of no relationship with age in the toddler years (Provasi \& Bobin-Bègue, 2003). The free drumming procedure employed in the current study is equally appropriate for use in early childhood, and it would be interesting for future studies using such a task to document when changes in the relationship between age and SMT occur, and explore the motor or cognitive skills, or experience, that motivate this change.

Our findings may suggest a contribution of passive experience to infant SMT. We find that tempo correlates with parent height, such that infants of taller parents show a slower SMT. Adult studies relating anthropometry to preferred tempo at which to move (Dahl et al., 2014; Mishima, 1965) could suggest that the link with body size is mediated by walking pace, with walking cadence providing a resonant tempo. However, the correlations found in prior studies could simply be a product of our own machinery, in that in a consistently proportioned body, a comfortable rate to move likely correlates across different motor actions. The current study is better suited to consider whether walking, as a predominant rhythmic action primes the rate of other rhythmic movements. Infants receive information, particularly vestibular information, at the rate of their mother's walking cadence, both in the womb and for months post-natally. Vestibular information is known to impact rhythm perception (Phillips-Silver \& Trainor, 2005, 2008; Trainor, Gao, Lei, Lehtovaara, \& Harris, 2009). We argue that the relationship we find between parent height and infant SMT may be the product of the vestibular experience infants receive while being carried.

Although we do not have strong evidence for the null, the lack of relationship between infant SMT and own body size is also consistent with the idea that it is experience of locomotion that is crucial. Aside from the fact that there was variability in the amount of self-locomotive experience between infants tested in the current study, the rapid physical growth and development of motoric skill within each infant would presumably provide each subject highly variable (a)rhythmic self-generated experience to draw upon. Infant walking does not show the regularity of adult walking, and regularity needs to be learned; above infant age or body size, amount of experience of walking is the best predictor of walking competency (Adolph, Vereijken, \& Shrout, 2003). Todd et al. (2007) suggest even small changes in body size at the tail end of adolescence may prevent a stable relationship between preferred beat rate and anthropometry. It is thusa plausible that the relationship between own body size 
and SMT may not appear until mid-adulthood, which is the point in the lifespan at which SMT seems to plateau (McAuley et al., 2006).

Due to the limited time and testing space we had with participants in this opportunity sample, we were unable to directly test whether parent cadence is indeed the factor underlying the relationship between parent height and infant SMT. Our proxy measure of parental cadence (parental height) most likely weakens the association that we report. In order to fully represent the infant experience, more precise data, directly measuring the time spent carried by the caregiver tested, the time spent carried by additional caregivers, and critically, the tempi of these experiences, are necessary. Such data to confirm our interpretation could be achieved through naturalistic day long diarized actigraphy measures, or via experimental manipulation of rate of carrying. Future work comparing pre- and post-natal experience of caregiver locomotion could give additional insight into how such pervasive early experience builds into infant rhythm perception and production. Finally, more in-depth investigation of the rhythmic predilections of infants as they master crawling and walking would also allow us to draw inference on how own motor experience might disrupt the relationship indicated in the current data between parent body size and infant rhythm.

Our study is the first to measure SMT from infants below 18 months of age. We developed a simple drumming task allowing data collection from infants as young as 5 months old that remains feasible to use over the entire lifespan. We find that infant drumming becomes faster and more regular with age, and reveal a relationship between infant SMT and parental height that we interpret as indicative of a contribution of the experience of being carried by the caregiver.

\section{ACKNOWLEDGMENTS}

This study was supported by an ESRC studentship awarded to SR. We are grateful to all of the families who took part in this study, and to the Wellcome Trust and Polka Theatre for hosting the BrainWaves Festival where data collection took place. We thank Giulia Ghillia, Iona Lamy-Yang, and Ana Skolaris for their assistance collecting and coding the data.

\section{CONFLICT OF INTEREST}

All authors report no conflict of interest.

\section{DATA AVAILABILITY STATEMENT}

Data are available on request from the authors. Contact Sinead Rocha on sineadrocha@gmail.com.

\section{ORCID}

Sinead Rocha (D) https://orcid.org/0000-0001-5231-9062

\section{REFERENCES}

Adolph, K. E., Vereijken, B., \& Shrout, P. E. (2003). What changes in infant walking and why. Child Development, 74(2), 475-497. https:// doi.org/10.1111/1467-8624.7402011
Bobin-Bègue, A., \& Provasi, J. (2008). Régulation rythmique avant 4 ans: Effet d'un tempo auditif sur le tempo moteur. L'année Psychologique, 108(04), 631-638.

Dahl, S., Huron, D., Brod, G., \& Altenmüller, E. (2014). Preferred dance tempo: Does sex or body morphology influence how we groove? Journal of New Music Research, 43(2), 214-223. https://doi. org/10.1080/09298215.2014.884144

Dienes, Z. (2014). Using Bayes to get the most out of non-significant results. Frontiers in Psychology, 5, 781. https://doi.org/10.3389/ fpsyg.2014.00781

Fraisse, P. (1982). Rhythm and tempo. In D. Deutsch (Ed.), The psychology of music (pp. 149-180). Orlando, FL: Academic Press.

Fujii, S., Watanabe, H., Oohashi, H., Hirashima, M., Nozaki, D., \& Taga, G. (2014). Precursors of dancing and singing to music in three-to four-months-old infants. PLoS One, 9(5), e97680. https://doi. org/10.1371/journal.pone.0097680

Goldfield, E. C. (1995). Emergent forms: Origins and early development of human action and perception. Oxford, UK: Oxford University Press.

Ilari, B. (2015). Rhythmic engagement with music in early childhood: A replication and extension. Journal of Research in Music Education, 62(4), 332-343. https://doi.org/10.1177/0022429414555984

Iversen, J. (2016). In the beginning was the beat: Evolutionary origins of musical rhythm in humans. In R. Hartenberger (Ed.), The Cambridge companion to percussion, (281-295). Cambridge, UK: Cambridge University Press.

McAuley, J. D., Jones, M. R., Holub, S., Johnston, H. M., \& Miller, N. S. (2006). The time of our lives: Life span development of timing and event tracking. Journal of Experimental Psychology: General, 135(3), 348-367. https://doi.org/10.1037/0096-3445.135.3.348

Mishima, J. (1965). Introduction to the morphology of human behavior. The experimental study of the mental tempo. Tokyo: Tokyo Publishing Co.

Phillips-Silver, J., \& Trainor, L. J. (2005). Feeling the beat: Movement influences infants' rhythm perception. Science, 308, 1430. https:// doi.org/10.1126/science.1110922

Phillips-Silver, J., \& Trainor, L. J. (2008). Vestibular influence on auditory metrical interpretation. Brain and Cognition, 67(1), 94-102.

Provasi, J., \& Bobin-Bègue, A. (2003). Spontaneous motor tempo and rhythmical synchronisation in $2 \frac{1}{2}$-and 4 -year-old children. International Journal of Behavioral Development, 27(3), 220-231. https://doi.org/10.1080/01650250244000290

Ravignani, A., Honing, H., \& Kotz, S. A. (2017). The evolution of rhythm cognition: Timing in music and speech. Frontiers in Human Neuroscience, 11, 303-303.

Repp, B. H. (2007). Embodied rhythm: commentary on" The Contribution of Anthropometric Factors to Individual Differences in the Perception of Rhythm" by Neil P. McAngus Todd, Rosanna Cousins, and Christopher S. Lee. Empirical Musicology Review, 2(1).

Rocha, S., \& Mareschal, D. (2017). Getting into the groove: The development of tempo-flexibility between 10 and 18 months of age. Infancy, 22(4), 540-551. https://doi.org/10.1111/infa.12169

Savage, P. E., Brown, S., Sakai, E., \& Currie, T. E. (2015). Statistical universals reveal the structures and functions of human music. Proceedings of the National Academy of Sciences of the United States of America, 112(29), 8987-8992. https://doi.org/10.1073/ pnas.1414495112

Thelen, E. (1979). Rhythmical stereotypies in normal human infants. Animal Behaviour, 27, 699-715. https://doi.org/10.1016/00033472(79)90006-X

Thelen, E. (1981). Rhythmical behavior in infancy: An ethological perspective. Developmental Psychology, 17(3), 237-258. https://doi. org/10.1037/0012-1649.17.3.237

Todd, N., Cousins, R., \& Lee, C. (2007). The contribution of anthropometric factors to individual differences in the perception of rhythm. Empirical Musicology Review, 2, 1-13. https://doi.org/10.18061/ $1811 / 24478$ 
Todd, N., \& Lee, C. (2007). Reply to "Embodied rhythm" by Bruno Repp and "Do preferred beat rate and entrainment to the beat have a common origin in movement?" by Laurel Trainor. Empirical Musicology Review, 2, 110-112. https://doi.org/10.18061/ $1811 / 28860$

Trainor, L. J. (2007). Do preferred beat rate and entrainment to the beat have a common origin in movement? Empirical Musicology Review, 2, 17-20. https://doi.org/10.18061/1811/24480

Trainor, L. J., Gao, X., Lei, J. J., Lehtovaara, K., \& Harris, L. R. (2009). The primal role of the vestibular system in determining musical rhythm. Cortex, 45(1), 35-43. https://doi.org/10.1016/j.cortex.2007.10.014

Vanneste, S., Pouthas, V., \& Wearden, J. H. (2001). Temporal control of rhythmic performance: A comparison between young and old adults. Experimental Aging Research, 27(1), 83-102. https://doi. org/10.1080/036107301750046151

Wickham, H. (2016). ggplot2: Elegant graphics for data analysis. New York: Springer-Verlag.
Zentner, M., \& Eerola, T. (2010). Rhythmic engagement with music in infancy. Proceedings of the National Academy of Sciences of the United States of America, 107(13), 5768-5773. https://doi.org/10.1073/ pnas. 1000121107

\section{SUPPORTING INFORMATION}

Additional supporting information may be found online in the Supporting Information section.

How to cite this article: Rocha S, Southgate V, Mareschal D. Infant Spontaneous Motor Tempo. Developmental Science. 2020;00:e13032. https://doi.org/10.1111/desc.13032 